\title{
Hypertension, Gender, Older Age, and Their Relationships with COVID-19 Mortality: Meta-Analysis
}

\author{
Annissa Devi Permata'), Bhisma Murti'), Didik Gunawan Tamtomo') \\ 1)Masters Program in Public Health, Universitas Sebelas Maret \\ 2)Faculty of Medicine, Universitas Sebelas Maret
}

\section{ABSTRACT}

Background: Coronavirus Disease 2019 (COVID-19) is an infectious disease caused by Severe Acute Respiratory Syndrome Coronavirus 2 (SARSCoV-2). SARS-CoV-2 is a new type of coronavirus that has never been previously identified in humans. Globally, 213 countries (as of August 11, 2020) are facing serious consequences from the ongoing COVID-19 pandemic. This study aimed to analyze the magnitude of the relationship of hypertension, gender, and older age to COVID-19 mortality with a meta-analysis study.

Subjects and Method: This was a systematic review and meta-analysis conducted by following the PRISMA flow diagram. The article search process is carried out through a journal database which includes: PubMed, Science Direct, Springer Link, and PMC Europe by selecting articles published in 2020-2021. The keywords used included: "sex" OR "gender" AND "older age" AND "hypertension" AND "mortality" OR "death" OR "fatal outcome" OR "Predictors outcome" OR "Impact" AND "coronavirus" OR "Covid-19" OR "SARS-COV-2" OR "2019 n-Cov" OR "severe acute respiratory syndrome related coronavirus". The inclusion criteria were full text articles with a retrospective cohort study design. The article is in English, and the analysis used is multivariate with adjusted Odds Ratio. Eligible articles were analyzed using the Revman $5.3 \mathrm{app}$.

Results: A total of 20 articles were reviewed in this study. A meta-analysis of 10 retrospective cohort studies showed that hypertension increased COVID-19 mortality by 1.40 times compared with no hypertension $(\mathrm{aOR}=1.40$; 95\% $\mathrm{CI}=1.04$ to 1.89 ; $\mathrm{p}=0.030$ ). A meta-analysis of 10 retrospective cohort studies showed that males had a 1.42 times increased risk of COVID-19 mortality compared to females $(\mathrm{aOR}=1.42 ; 95 \% \mathrm{CI}=1.20$ to $1.67 ; \mathrm{p}<0.001)$. Meta-analysis of 7 retrospective cohort studies showed that old age has a 3.42 times increased risk of COVID-19 death compared to younger age $(\mathrm{aOR}=4.15 ; 95 \% \mathrm{CI}=2.35$ to $7.32 ; \mathrm{p}$ $<0.001)$.

Conclusion: Hypertension, gender, and old age increase the risk of dying from COVID-19.

Keywords: Hypertension, Gender, Old Age, COVID-19 Death, Meta-analysis

\section{Correspondence:}

Annissa Devi Permata. Masters Program in Public Health, Universitas Sebelas Maret. Jl. Ir. Sutami, 36A, Surakarta 57126, Central Java, Indonesia. Email: 96annissadevipermata@gmail.com

Cite this as:

Permata AD, Murti B, Tamtomo DG (2021). Hypertension, Gender, Older Age, and Their Relationships with COVID-19 Mortality: Meta-Analysis. J Epidemiol Public Health. 06(01): 98-111. https://doi.org/10.26911/jepublichealth.2021.06.01.10.

(c) (i) (-) Journal of Maternal and Child Health is licensed under a Creative Commons Attribution-NonCommercial-ShareAlike 4.o International License.

\section{BACKGROUND}

Coronavirus Disease (COVID-19) was first documented in Wuhan City, Hubei Province, China in December 2019 as an outbreak of pneumonia. Based on established phylogeny, taxonomy and practice, on
February 11, 2020, the World Health Organization (WHO) named the disease COVID19, WHO declared COVID-19 a global emergency on January 30, 2020 and as a pandemic on March 11, 2020. Globally, 213 countries (as of 11 August 2020) are facing 
serious consequences from the ongoing COVID-19 pandemic (Eslam et al., 2020).

Based on the World Health Organization (WHO), as of February 6, there were 104,370,550 confirmed cases and 2,271,180 deaths due to COVID-19 worldwide (CDC, 2020). The current standard of care test for diagnosing COVID-19 is to collect a nasopharyngeal swab (NP) and use a reverse transcription polymerase chain reaction (RT-PCR) assay to detect SARS-CoV-2 RNA (Severe Acute Respiratory Syndrome Coronavirus-2 Ribonucleic Acid). Investigation of risk factors for mortality with COVID-19 in patients has mostly focused on patient characteristics such as older age, gender, and comorbidities (Magleby, 2020).

Comorbid patients with COVID-19 have a high risk of disease severity, ICU admission, including death. Hypertension is the most common comorbidity among COVID-19 patients and the prevalence of death among hypertensive patients due to COVID-19 is 58.3\% (Musharrat, 2020). Global data also shows a higher COVID-19 case fatality rate among men than women. Most of the few countries for which data are available show a male to female case fatality ratio higher than 1.0, ranging up to 3.5 in some cases (Dehingia et al., 2020). Old age also has a higher mortality rate. About $80 \%$ of deaths have occurred in patients aged > 60 years in South Korea and Italy (Kang et al., 2020).

Research in the Federal Capital Territory (FCT) state showed that significant risk factors were associated with COVID-19 mortality in older age and male gender, progressively the older age group experienced an increase of about 51 times and in men 78\% higher than women in COVID-19 mortality (Elimian et al., 2020).

COVID-19 mortality and outcomes differ significantly around the world. Pakistan has passed the peak of the pande- mic. Pakistan's death rate of $2.13 \%$ is significantly lower than that reported in the Americas and Europe and lower than that of its neighbors Iran, Afghanistan and China, but comparable to India. This study reported deaths by gender, old age and hypertension (Nasir et al., 2020).

During the COVID-19 pandemic, various studies have been carried out to see the relationship between hypertension, gender and old age with COVID-19 mortality, but the results of the research still do not show consistent results. Further analysis is needed to arrive at a convincing conclusion. Therefore, this study may help further in understanding the results of the collection of COVID-19 deaths, particularly the association with hypertension, gender, and old age with COVID-19 mortality. Researchers use a systematic review approach to relevant studies by conducting meta-analysis.

\section{SUBJECTS AND METHOD}

\section{Study Design}

This study is a systematic review and metaanalysis. The articles used in this study were obtained from several databases, including PubMed, Science Direct, Springer Link, and PMC Europe. The keywords to search for articles were as follows: "sex" OR "gender" AND "older age" AND "hypertension" AND "mortality" OR "death" OR "fatal outcome" OR "Predictors outcome" OR "Impact" AND "coronavirus" OR "Covid-19" OR "SARS-COV-2" OR "2O19 nCov" OR "severe acute respiratory syndrome related coronavirus".

\section{Inclusion Criteria}

Articles included in this study must be full papers. Titles that match hypertension, gender, and old age are associated with death in COVID-19 patients, Articles selected for old age are with age characteristics $>50$ years, articles that use the design retrospective cohort, article publish- 
ed in English, the analysis used was multivariate by ascertaining the adjusted odds ratio (aOR).

\section{Exclusion Criteria}

The articles excluded in this study were the sample in articles $\mathrm{n}<100$, and not multivariate analysis studies, mixed studies such as stroke, cerebrospinal hemorrhage, chronic liver disease and others.

\section{Operational Definition of Variables} The population in the study included COVID-19 patients with intervention in the form of hypertension, gender and old age.

The comparison was not hypertension, female gender and not old age and outcomes in the form of COVID-19 death.

COVID-19 is a disease that has shown serious infection or severe symptoms causing death caused by Severe Acute Respiratory Syndrome Coronavirus 2 (SARSCoV-2).

Hypertension is a major cardiovascular disease and premature death, due to increased intravascular volume as one of the risks of COVID-19 death.
Gender is the biological difference between women and men as one of the risks of death from COVID-19

Old age is a phase of decreasing physical ability and susceptibility to infection as a person ages as one of the risks of COVID19 death.

\section{Data Analysis}

Data processing was carried out by Review Manager (RevMan 5.3) by calculating effect size and heterogeneity to determine the combined research model and form the final result of the meta-analysis.

\section{RESULTS}

The article review process can be seen in the PRISMA flow diagram in Figure 1. Figure 2 shows the areas where articles were taken according to the inclusion criteria. This meta-analysis analyzed 3 articles from Africa, 8 from North America, 4 from Asia, and 5 from Europe.

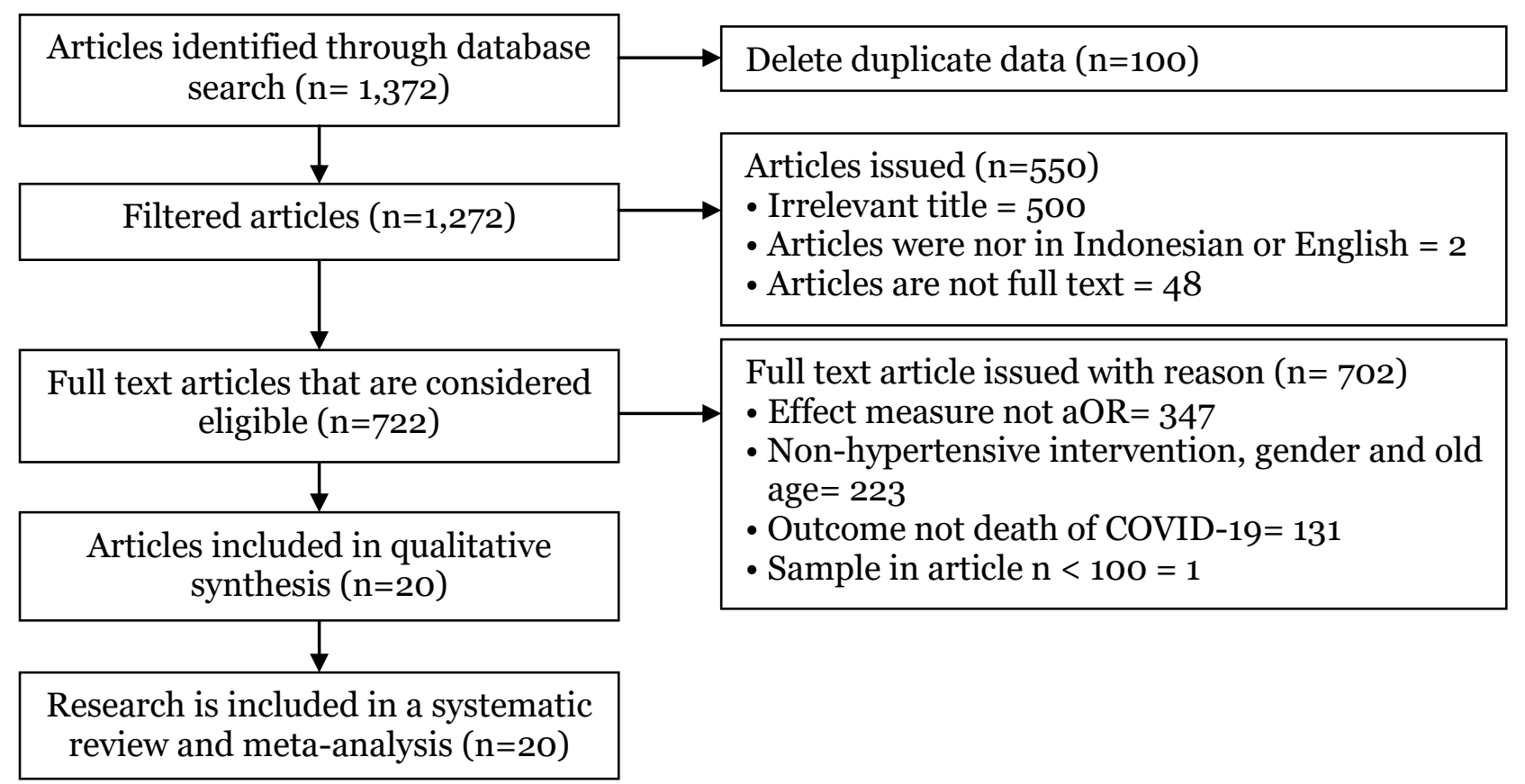

Figure 1. PRISMA Flow Diagram 


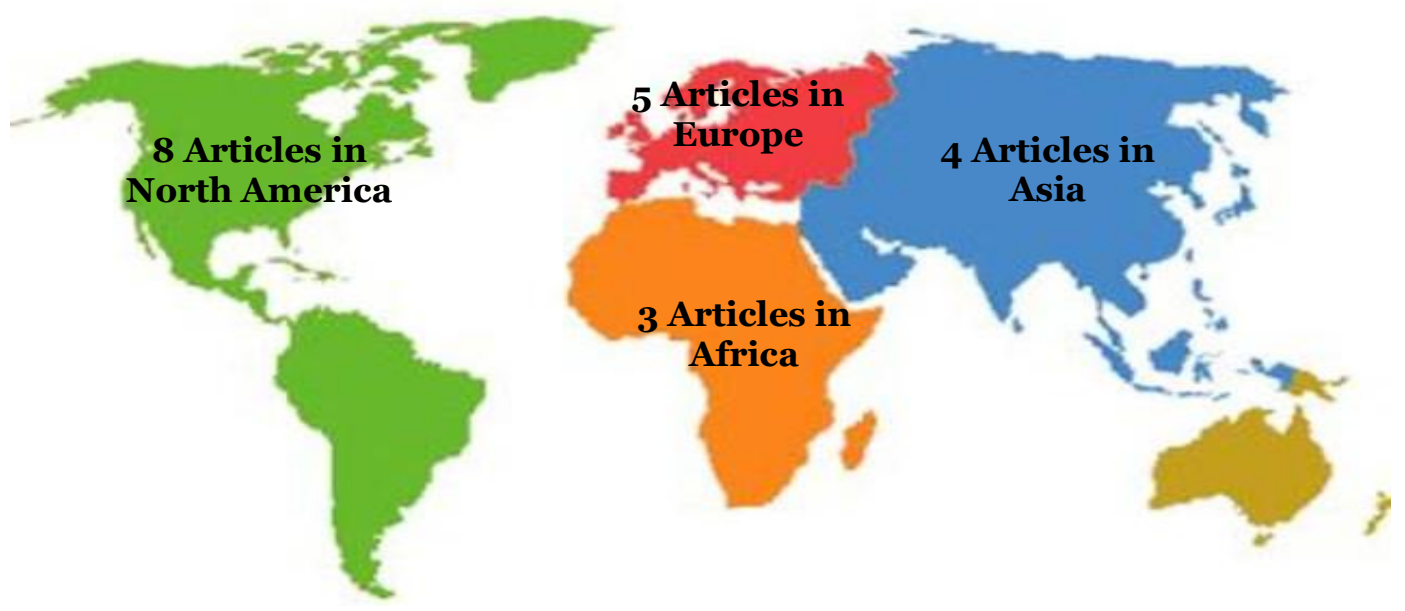

Figure 2. Distribution of Primary Studies by Continent

(Buzan Tony, 2021)

The assessment of the quality of the primary studies was carried out quantitatively and qualitatively which can be seen in Table 1, Table 2 and Table 3 below. This research was conducted using the Critical Appraisal Checklist for Cohort Study sourced from the Center for Evidence Based Management (CEBMa, 2014).

Table 1. Quality Assessment of Hypertension Articles: Retrospective Cohort Study

\begin{tabular}{|c|c|c|c|c|c|c|c|c|c|c|c|c|c|}
\hline \multirow{2}{*}{ Primary Study } & \multicolumn{12}{|c|}{ Criteria } & \multirow{2}{*}{ Total } \\
\hline & $\mathbf{1}$ & 2 & 3 & 4 & 5 & 6 & 7 & 8 & 9 & 10 & $\mathbf{1 1}$ & 12 & \\
\hline Bourgne et al. (2021) & 1 & 1 & 1 & 1 & 1 & 1 & 1 & 1 & 1 & 1 & 1 & 1 & 12 \\
\hline Hashemi et al. (2020) & 1 & 1 & 1 & 1 & 1 & 1 & 1 & $\mathrm{O}$ & 1 & 1 & 1 & 1 & 11 \\
\hline Hoobs et al. (2021) & 1 & 1 & 1 & O & 1 & 1 & 1 & $\mathrm{O}$ & 1 & 1 & 1 & 1 & 10 \\
\hline Leulseged et al. (2021) & 1 & 1 & 1 & 1 & 1 & 1 & 1 & $\mathrm{O}$ & 1 & 1 & 1 & 1 & 11 \\
\hline Magleby et al. (2020) & 1 & 1 & 1 & 1 & 1 & 1 & 1 & 1 & 1 & 1 & 1 & 1 & 12 \\
\hline Osibagun et al. (2021) & 1 & 1 & 1 & 1 & 1 & 1 & 1 & o & 1 & 1 & 1 & 1 & 11 \\
\hline Rosental et al. (2021) & 1 & 1 & 1 & 1 & 1 & 1 & 1 & 1 & 1 & 1 & 1 & 1 & 12 \\
\hline Surendra et al. (2021) & 1 & 1 & 1 & 1 & 1 & 1 & 1 & 1 & 1 & 1 & 1 & 1 & 12 \\
\hline Walker et al. (2021) & 1 & 1 & 1 & $\mathrm{O}$ & 1 & 1 & 1 & $\mathrm{O}$ & 1 & 1 & 1 & 1 & 10 \\
\hline Yip et al. (2021) & 1 & 1 & 1 & 1 & 1 & $\mathrm{O}$ & 1 & $\mathrm{O}$ & 1 & 1 & 1 & 1 & 10 \\
\hline
\end{tabular}

Answer: 1 = Yes and $0=$ No

Table 2. Assessment of Article Quality Gender: Retrospective Cohort Study

\begin{tabular}{|c|c|c|c|c|c|c|c|c|c|c|c|c|c|}
\hline \multirow{2}{*}{ Primary Study } & \multicolumn{12}{|c|}{ Criteria } & \multirow{2}{*}{ Total } \\
\hline & $\mathbf{1}$ & 2 & 3 & 4 & 5 & 6 & 7 & 8 & 9 & 10 & $\mathbf{1 1}$ & 12 & \\
\hline Elimian et al. (2021) & 1 & 1 & 1 & $\mathrm{O}$ & 1 & 1 & 1 & $\mathrm{O}$ & 1 & 1 & 1 & 1 & 10 \\
\hline Guzman et al. (2020) & 1 & 1 & 1 & 1 & 1 & 1 & 1 & 1 & 1 & 1 & 1 & 1 & 12 \\
\hline Harisson et al. (2020) & 1 & 1 & 1 & 1 & 1 & 1 & 1 & $\mathrm{O}$ & 1 & 1 & 1 & 1 & 11 \\
\hline Hashemi et al. (2020) & 1 & 1 & 1 & 1 & 1 & 1 & 1 & $\mathrm{O}$ & 1 & 1 & 1 & 1 & 11 \\
\hline Hoobs et al. (2021) & 1 & 1 & 1 & O & 1 & 1 & 1 & $\mathrm{O}$ & 1 & 1 & 1 & 1 & 10 \\
\hline Leulseged et al. (2021) & 1 & 1 & 1 & 1 & 1 & 1 & 1 & 0 & 1 & 1 & 1 & 1 & 11 \\
\hline Manuel et al. (2020) & 1 & 1 & 1 & $\mathrm{O}$ & 1 & 1 & 1 & 1 & 1 & 1 & 1 & 1 & 11 \\
\hline Rossa et al. (2021) & 1 & 1 & 1 & 1 & 1 & 1 & 1 & 0 & 1 & 1 & 1 & 1 & 11 \\
\hline Rosental et al. (2021) & 1 & 1 & 1 & 1 & 1 & 1 & 1 & 1 & 1 & 1 & 1 & 1 & 12 \\
\hline Surendra et al. (2021) & 1 & 1 & 1 & 1 & 1 & 1 & 1 & 1 & 1 & 1 & 1 & 1 & 12 \\
\hline
\end{tabular}

Answer: $1=$ Yes and $0=$ No 
Permata et al./ Hypertension, Gender, Older Age, and COVID-19 Mortality

Table 3. Quality Assessment of Old Age Articles: Retrospective Cohort Study

\begin{tabular}{|c|c|c|c|c|c|c|c|c|c|c|c|c|c|}
\hline \multirow{2}{*}{ Primary Study } & \multicolumn{12}{|c|}{ Criteria } & \multirow{2}{*}{ Total } \\
\hline & $\mathbf{1}$ & 2 & 3 & 4 & 5 & 6 & 7 & 8 & 9 & 10 & $\mathbf{1 1}$ & 12 & \\
\hline Hoobs et al. (2021) & 1 & 1 & 1 & O & 1 & 1 & 1 & 0 & 1 & 1 & 1 & 1 & 10 \\
\hline Imam et al. (2020) & 1 & 1 & 1 & 1 & 1 & 1 & 1 & 1 & 1 & 1 & 1 & 1 & 12 \\
\hline Islam et al. (2020) & 1 & 1 & 1 & 1 & 1 & 1 & 1 & 0 & 1 & 1 & 1 & 1 & 11 \\
\hline Leulseged et al. (2021) & 1 & 1 & 1 & 1 & 1 & 1 & 1 & 0 & 1 & 1 & 1 & 1 & 11 \\
\hline Yahui et al. (2021) & 1 & 1 & 1 & 1 & 1 & 1 & 1 & o & 1 & 1 & 1 & 1 & 11 \\
\hline Cedano et al. (2020) & 1 & 1 & 1 & 1 & 1 & 1 & 1 & $\mathrm{O}$ & 1 & 1 & 1 & 1 & 11 \\
\hline Gerwen et al. (2020) & 1 & 1 & 1 & $\mathrm{O}$ & 1 & 1 & 1 & $\mathrm{O}$ & 1 & 1 & 1 & 1 & 10 \\
\hline
\end{tabular}

Answer: $1=$ Yes and $0=$ No

Based on the assessment of the quality of the primary articles above, the article quality scores are 10 to 11 . This indicates that the articles have good quality for meta-analysis (CEBMa, 2014).

Table 4. Description of the primary studies of hypertension included in the metaanalysis

\begin{tabular}{|c|c|c|c|c|c|c|c|c|}
\hline No & $\begin{array}{l}\text { Author } \\
\text { (Year) }\end{array}$ & $\begin{array}{c}\text { Country } \\
\text { (Conti- } \\
\text { nent) }\end{array}$ & $\begin{array}{l}\text { Study } \\
\text { Design }\end{array}$ & $\begin{array}{c}\text { Sam } \\
\text { ple }\end{array}$ & $\begin{array}{c}\mathbf{P} \\
\text { (Popula- } \\
\text { tion) }\end{array}$ & $\begin{array}{c}\text { I } \\
\text { (Inter- } \\
\text { vention) }\end{array}$ & $\begin{array}{c}\mathrm{C} \\
\text { (Compa- } \\
\text { rison) }\end{array}$ & $\begin{array}{c}\text { O } \\
\text { (Out- } \\
\text { come) }\end{array}$ \\
\hline 1 & $\begin{array}{l}\text { Bourgne } \\
\text { et al } \\
(2021)\end{array}$ & $\begin{array}{l}\text { France } \\
\text { (Europe) }\end{array}$ & $\begin{array}{l}\text { Retrospective } \\
\text { Cohort Study }\end{array}$ & 287 & $\begin{array}{l}\text { COVID-19 } \\
\text { patient }\end{array}$ & $\begin{array}{l}\text { Hyper- } \\
\text { tension }\end{array}$ & $\begin{array}{l}\text { Not } \\
\text { Hyperten } \\
\text { sion }\end{array}$ & $\begin{array}{l}\text { COVID-19 } \\
\text { death }\end{array}$ \\
\hline 2 & $\begin{array}{l}\text { Hashemi } \\
\text { et al. } \\
(2020)\end{array}$ & $\begin{array}{l}\text { USA } \\
\text { (North } \\
\text { America) }\end{array}$ & $\begin{array}{l}\text { Retrospective } \\
\text { Cohort Study }\end{array}$ & 363 & $\begin{array}{l}\text { COVID-19 } \\
\text { patient }\end{array}$ & $\begin{array}{l}\text { Hyperten } \\
\text { sion }\end{array}$ & $\begin{array}{l}\text { Not } \\
\text { Hyperten } \\
\text { sion }\end{array}$ & $\begin{array}{l}\text { COVID-19 } \\
\text { death }\end{array}$ \\
\hline 3 & $\begin{array}{l}\text { Hoobs et } \\
\text { al (2021) }\end{array}$ & $\begin{array}{l}\text { USA } \\
\text { (North } \\
\text { America) }\end{array}$ & $\begin{array}{l}\text { Retrospective } \\
\text { Cohort Study }\end{array}$ & 502 & $\begin{array}{l}\text { COVID-19 } \\
\text { patient }\end{array}$ & $\begin{array}{l}\text { Hyperten } \\
\text { sion }\end{array}$ & $\begin{array}{l}\text { Not } \\
\text { Hyperten } \\
\text { sion }\end{array}$ & $\begin{array}{l}\text { COVID-19 } \\
\text { death }\end{array}$ \\
\hline 4 & $\begin{array}{l}\text { Leulseged } \\
\text { et al. } \\
(2021)\end{array}$ & $\begin{array}{l}\text { Ethiopia } \\
\text { (Africa) }\end{array}$ & $\begin{array}{l}\text { Retrospective } \\
\text { Cohort Study }\end{array}$ & 429 & $\begin{array}{l}\text { COVID-19 } \\
\text { patient }\end{array}$ & $\begin{array}{l}\text { Hyperten } \\
\text { sion }\end{array}$ & $\begin{array}{l}\text { Not } \\
\text { Hyperten } \\
\text { sion }\end{array}$ & $\begin{array}{l}\text { COVID-19 } \\
\text { death }\end{array}$ \\
\hline 5 & $\begin{array}{l}\text { Magleby } \\
\text { et al. } \\
(2020)\end{array}$ & $\begin{array}{l}\text { USA } \\
\text { (North } \\
\text { America) }\end{array}$ & $\begin{array}{l}\text { Retrospective } \\
\text { Cohort Study }\end{array}$ & 678 & $\begin{array}{l}\text { COVID-19 } \\
\text { patient }\end{array}$ & $\begin{array}{l}\text { Hyperten } \\
\text { sion }\end{array}$ & $\begin{array}{l}\text { Not } \\
\text { Hyperten } \\
\text { sion }\end{array}$ & $\begin{array}{l}\text { COVID-19 } \\
\text { death }\end{array}$ \\
\hline 6 & $\begin{array}{l}\text { Osibagun } \\
\text { et al. } \\
(2021)\end{array}$ & $\begin{array}{l}\text { Nigeria } \\
\text { (Africa) }\end{array}$ & $\begin{array}{l}\text { Retrospective } \\
\text { Cohort Study }\end{array}$ & 2184 & $\begin{array}{l}\text { COVID-19 } \\
\text { patient }\end{array}$ & $\begin{array}{l}\text { Hyperten } \\
\text { sion }\end{array}$ & $\begin{array}{l}\text { Not } \\
\text { Hyperten } \\
\text { sion }\end{array}$ & $\begin{array}{l}\text { COVID-19 } \\
\text { death }\end{array}$ \\
\hline 7 & $\begin{array}{l}\text { Rosental } \\
\text { et al } \\
(2021)\end{array}$ & $\begin{array}{l}\text { USA } \\
\text { (North } \\
\text { America) }\end{array}$ & $\begin{array}{l}\text { Retrospective } \\
\text { Cohort Study }\end{array}$ & $\begin{array}{c}6478 \\
1\end{array}$ & $\begin{array}{l}\text { COVID-19 } \\
\text { patient }\end{array}$ & $\begin{array}{l}\text { Hyperten } \\
\text { sion }\end{array}$ & $\begin{array}{l}\text { Not } \\
\text { Hyperten } \\
\text { sion }\end{array}$ & $\begin{array}{l}\text { COVID-19 } \\
\text { death }\end{array}$ \\
\hline 8 & $\begin{array}{l}\text { Surendra } \\
\text { et al. } \\
(2021)\end{array}$ & $\begin{array}{l}\text { Indonesia } \\
\text { (Asia) }\end{array}$ & $\begin{array}{l}\text { Retrospective } \\
\text { Cohort Study }\end{array}$ & 4265 & $\begin{array}{l}\text { COVID-19 } \\
\text { patient }\end{array}$ & $\begin{array}{l}\text { Hyperten } \\
\text { sion }\end{array}$ & $\begin{array}{l}\text { Not } \\
\text { Hyperten } \\
\text { sion }\end{array}$ & $\begin{array}{l}\text { COVID-19 } \\
\text { death }\end{array}$ \\
\hline 9 & $\begin{array}{l}\text { Walker et } \\
\text { al. (2021) }\end{array}$ & $\begin{array}{l}\text { UK } \\
\text { (Eropa) }\end{array}$ & $\begin{array}{l}\text { Retrospective } \\
\text { Cohort Study }\end{array}$ & 347 & $\begin{array}{l}\text { COVID-19 } \\
\text { patient }\end{array}$ & $\begin{array}{l}\text { Hyperten } \\
\text { sion }\end{array}$ & $\begin{array}{l}\text { Not } \\
\text { Hyperten } \\
\text { sion }\end{array}$ & $\begin{array}{l}\text { COVID-19 } \\
\text { death }\end{array}$ \\
\hline 10 & $\begin{array}{l}\text { Yip et al. } \\
(2021)\end{array}$ & $\begin{array}{l}\text { Hongkon } \\
\text { g (Asia) }\end{array}$ & $\begin{array}{l}\text { Retrospective } \\
\text { Cohort Study }\end{array}$ & 1040 & $\begin{array}{l}\text { COVID-19 } \\
\text { patient }\end{array}$ & $\begin{array}{l}\text { Hyperten } \\
\text { sion }\end{array}$ & $\begin{array}{l}\text { Not } \\
\text { Hyperten } \\
\text { sion }\end{array}$ & $\begin{array}{l}\text { COVID-19 } \\
\text { death }\end{array}$ \\
\hline
\end{tabular}


Permata et al./ Hypertension, Gender, Older Age, and COVID-19 Mortality

\section{Results of the Relationship between Hypertension and Covid-19 Death Odis Ratio \\ Oclis Ratio \\ Stucly or Sulugroul) $\quad \log [0$ odls Ratio] SE Weight N, Ranclom, 95\% Cl \\ IV, Ranclom, 95\% Cl}

$\begin{array}{lrrrr}\text { Borgne 2021 } & -0.2357 & 0.4454 & 6.9 \% & 0.79[0.33,1.89] \\ \text { Hashemi 2020 } & 0.7885 & 0.4675 & 6.6 \% & 2.20[0.88,5.50] \\ \text { Hoobs 2021 } & -0.1744 & 0.3537 & 8.9 \% & 0.84[0.42,1.68] \\ \text { Leulseged 2021 } & 0.4492 & 0.4569 & 6.7 \% & 1.57[0.64,3.84] \\ \text { Magleby 2020 } & -0.3011 & 0.3013 & 10.2 \% & 0.74[0.41,1.34] \\ \text { Osibogun 2021 } & 1.4929 & 0.2801 & 10.8 \% & 4.45[2.57,7.71] \\ \text { Rosental 2021 } & 0.077 & 0.0444 & 16.8 \% & 1.08[0.99,1.18] \\ \text { Surendra 2021 } & 0.4121 & 0.1215 & 15.4 \% & 1.51[1.19,1.92] \\ \text { Walker 2021 } & 0.1484 & 0.3537 & 8.9 \% & 1.16[0.58,2.32] \\ \text { Yip 2020 } & 0.7885 & 0.363 & 8.7 \% & 2.20[1.08,4.48]\end{array}$

Heterogeneity: Tau $^{2}=0.14 ; \mathrm{Ch}^{2}=39.55, \mathrm{df}=9(\mathrm{P}<0.00001) ;{ }^{2}=77 \%$

Test for overall effect: $Z=2.22(P=0.03)$

$100.0 \% \quad 1.40[1.04,1.89]$

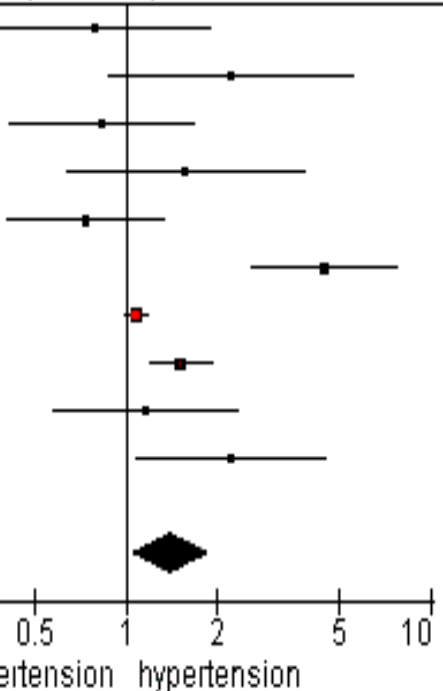

Figure 3. Forest plot of the relationship between hypertension and Covid-19 mortality

Based on the results of the forest plot (Figure 3). The results of a meta-analysis of a retrospective cohort study showed that hypertension increased the incidence of death in COVID-19 patients by 1.40 times compared to non-hypertensive $(\mathrm{p}=0.030)$. The heterogeneity of the research data shows $\mathrm{I}^{2}=77 \%$, so that the distribution of the data is declared heterogeneous (random effect model).

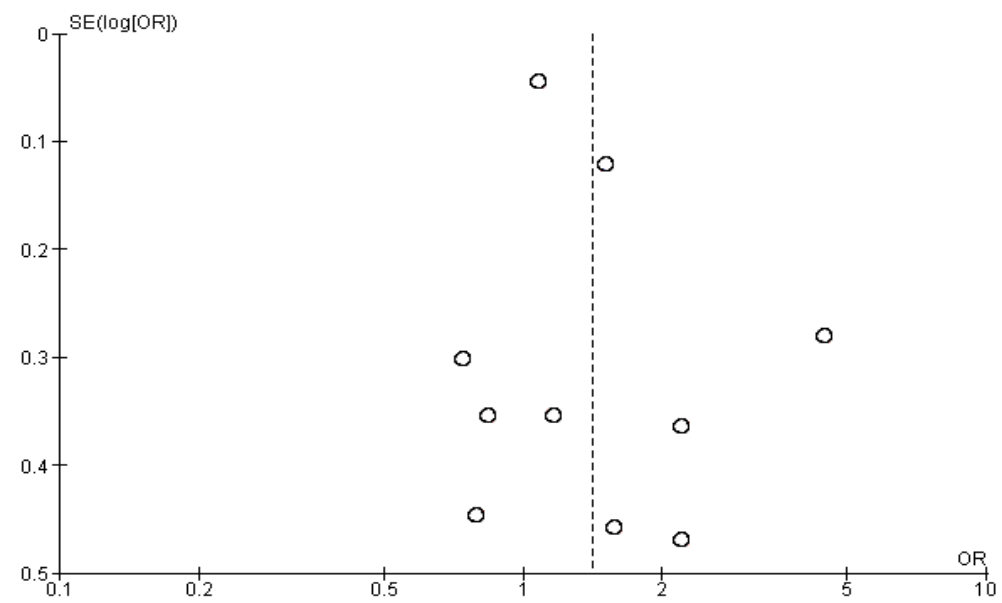

Figure 4. Funnel plot of the relationship between hypertension and Covid-19 mortality

The funnel plot (Figure 4) shows no publication bias as indicated by the plots on the right and left sides being symmetrical to each other and not forming an inverted funnel, where 5 plots are on the right and 5 plots are on the left. The plot on the left of the graph appears to have a standard error between 0 and 0.5 and the plot on the right has a standard error between 0.1 and 0.5 . 
Permata et al./ Hypertension, Gender, Older Age, and COVID-19 Mortality

Table 5. Description of the primary studies by gender included in the meta-analysis

\begin{tabular}{|c|c|c|c|c|c|c|c|c|}
\hline $\begin{array}{l}\mathbf{N} \\
\mathbf{o}\end{array}$ & $\begin{array}{l}\text { Author } \\
\text { (Year) }\end{array}$ & Country & $\begin{array}{c}\text { Study } \\
\text { Design }\end{array}$ & $\begin{array}{c}\text { Sam- } \\
\text { ple }\end{array}$ & $\begin{array}{c}\mathbf{P} \\
\text { (Popu- } \\
\text { lation) } \\
\end{array}$ & $\begin{array}{c}I \\
\begin{array}{c}\text { (Interven- } \\
\text { tion) }\end{array}\end{array}$ & $\begin{array}{c}\mathrm{C} \\
\text { (Compa- } \\
\text { rison) }\end{array}$ & $\begin{array}{c}\text { O } \\
\text { (Out- } \\
\text { come) } \\
\end{array}$ \\
\hline 1 & $\begin{array}{l}\text { Elimian } \\
\text { et al } \\
(2021)\end{array}$ & Nigerian & $\begin{array}{l}\text { Retrospec- } \\
\text { tive Cohort } \\
\text { Study }\end{array}$ & 36,496 & $\begin{array}{l}\text { COVID-19 } \\
\text { patient }\end{array}$ & Male & Female & $\begin{array}{l}\text { COVID-19 } \\
\text { mortality }\end{array}$ \\
\hline 2 & $\begin{array}{l}\text { Guzman } \\
\text { et al. } \\
(2020)\end{array}$ & England & $\begin{array}{l}\text { Retrospecti } \\
\text { ve Cohort } \\
\text { Study }\end{array}$ & 347 & $\begin{array}{l}\text { COVID-19 } \\
\text { patient }\end{array}$ & Male & Female & $\begin{array}{l}\text { COVID-19 } \\
\text { mortality }\end{array}$ \\
\hline 3 & $\begin{array}{l}\text { Harisson } \\
\text { et al. } \\
\text { (2020) }\end{array}$ & $\begin{array}{l}\text { United } \\
\text { States }\end{array}$ & $\begin{array}{l}\text { Retrospecti } \\
\text { ve Cohort } \\
\text { Study }\end{array}$ & 31461 & $\begin{array}{l}\text { COVID-19 } \\
\text { patient }\end{array}$ & Male & Female & $\begin{array}{l}\text { COVID-19 } \\
\text { mortality }\end{array}$ \\
\hline 4 & $\begin{array}{l}\text { Hashemi } \\
\text { et al. } \\
\text { (2020) }\end{array}$ & $\begin{array}{l}\text { United } \\
\text { States }\end{array}$ & $\begin{array}{l}\text { Retrospecti } \\
\text { ve Cohort } \\
\text { Study }\end{array}$ & 363 & $\begin{array}{l}\text { COVID-19 } \\
\text { patient }\end{array}$ & Male & Female & $\begin{array}{l}\text { COVID-19 } \\
\text { mortality }\end{array}$ \\
\hline 5 & $\begin{array}{l}\text { Hoobs et } \\
\text { al (2021) }\end{array}$ & $\begin{array}{l}\text { United } \\
\text { States) }\end{array}$ & $\begin{array}{l}\text { Retrospecti } \\
\text { ve Cohort } \\
\text { Study }\end{array}$ & 502 & $\begin{array}{l}\text { COVID-19 } \\
\text { patient }\end{array}$ & Male & Female & $\begin{array}{l}\text { COVID-19 } \\
\text { mortality }\end{array}$ \\
\hline 6 & $\begin{array}{l}\text { Leulsege } \\
\text { d et al. } \\
\text { (2021) }\end{array}$ & Ethiopia & $\begin{array}{l}\text { Retrospecti } \\
\text { ve Cohort } \\
\text { Study }\end{array}$ & 429 & $\begin{array}{l}\text { COVID-19 } \\
\text { patient }\end{array}$ & Male & Female & $\begin{array}{l}\text { COVID-19 } \\
\text { mortality }\end{array}$ \\
\hline 7 & $\begin{array}{l}\text { Manuel } \\
\text { et al } \\
(2020)\end{array}$ & Spain & $\begin{array}{l}\text { Retrospecti } \\
\text { ve Cohort } \\
\text { Study }\end{array}$ & 272 & $\begin{array}{l}\text { COVID-19 } \\
\text { patient }\end{array}$ & Male & Female & $\begin{array}{l}\text { COVID-19 } \\
\text { mortality }\end{array}$ \\
\hline 8 & $\begin{array}{l}\text { Rossa et } \\
\text { al. } \\
(2021)\end{array}$ & Italy & $\begin{array}{l}\text { Retrospecti } \\
\text { ve Cohort } \\
\text { Study }\end{array}$ & 1538 & $\begin{array}{l}\text { COVID-19 } \\
\text { patient }\end{array}$ & Male & Female & $\begin{array}{l}\text { COVID-19 } \\
\text { mortality }\end{array}$ \\
\hline 9 & $\begin{array}{l}\text { Rosental } \\
\text { et al. } \\
\text { (2021) }\end{array}$ & $\begin{array}{l}\text { United } \\
\text { States }\end{array}$ & $\begin{array}{l}\text { Retrospecti } \\
\text { ve Cohort } \\
\text { Study }\end{array}$ & 64781 & $\begin{array}{l}\text { COVID-19 } \\
\text { patient }\end{array}$ & Male & Female & $\begin{array}{l}\text { COVID-19 } \\
\text { mortality }\end{array}$ \\
\hline $\begin{array}{l}1 \\
0\end{array}$ & $\begin{array}{l}\text { Surendra } \\
\text { et al. } \\
(2021)\end{array}$ & Indonesia & $\begin{array}{l}\text { Retrospecti } \\
\text { ve Cohort } \\
\text { Study }\end{array}$ & 4265 & $\begin{array}{l}\text { COVID-19 } \\
\text { patient }\end{array}$ & Male & Female & $\begin{array}{l}\text { COVID-19 } \\
\text { mortality }\end{array}$ \\
\hline
\end{tabular}

\section{Results of the Relationship of Gender with Covid-19 Deaths}

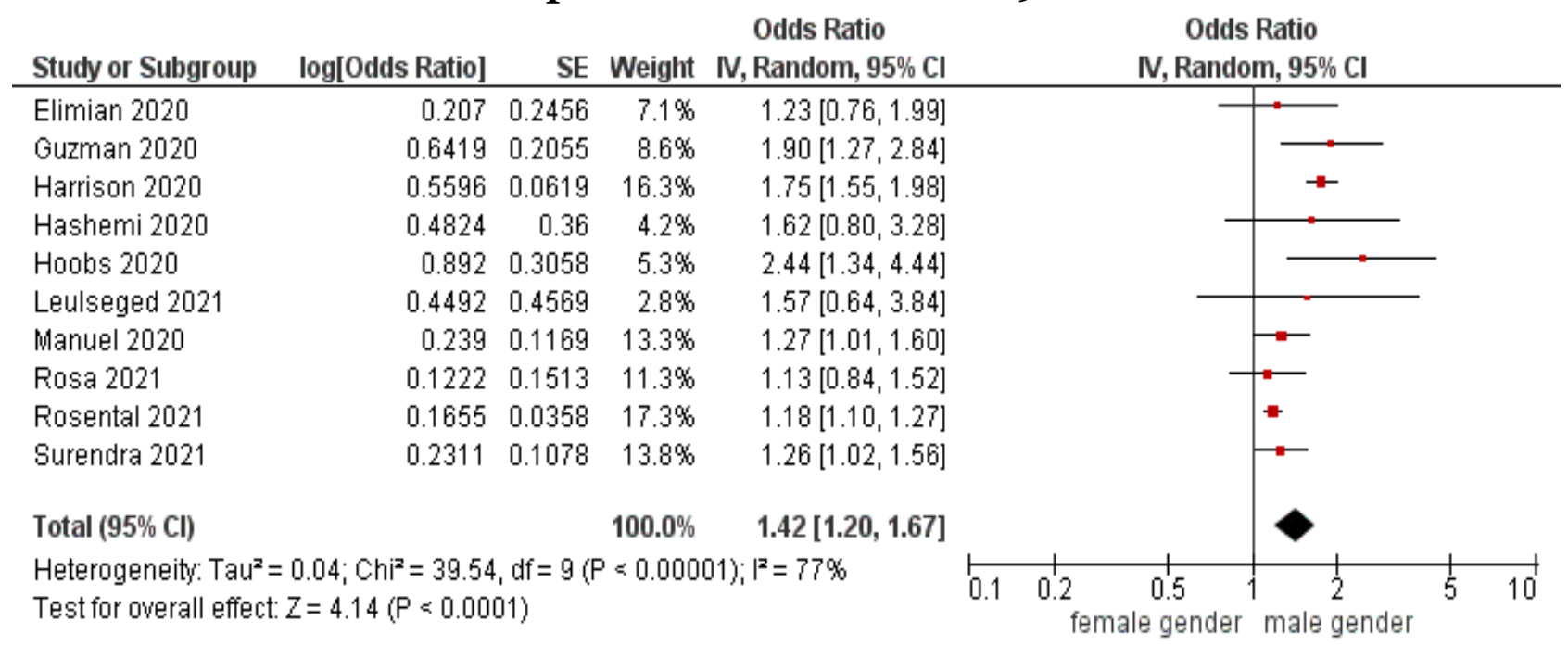

Figure 5. Forest plot of the relationship between gender and Covid-19 deaths 
Based on the results of the forest plot (Figure 5) The results of the meta-analysis of retrospective cohort studies showed that gender increased the incidence of death in COVID-19 patients by 1.42 times compared to female sex

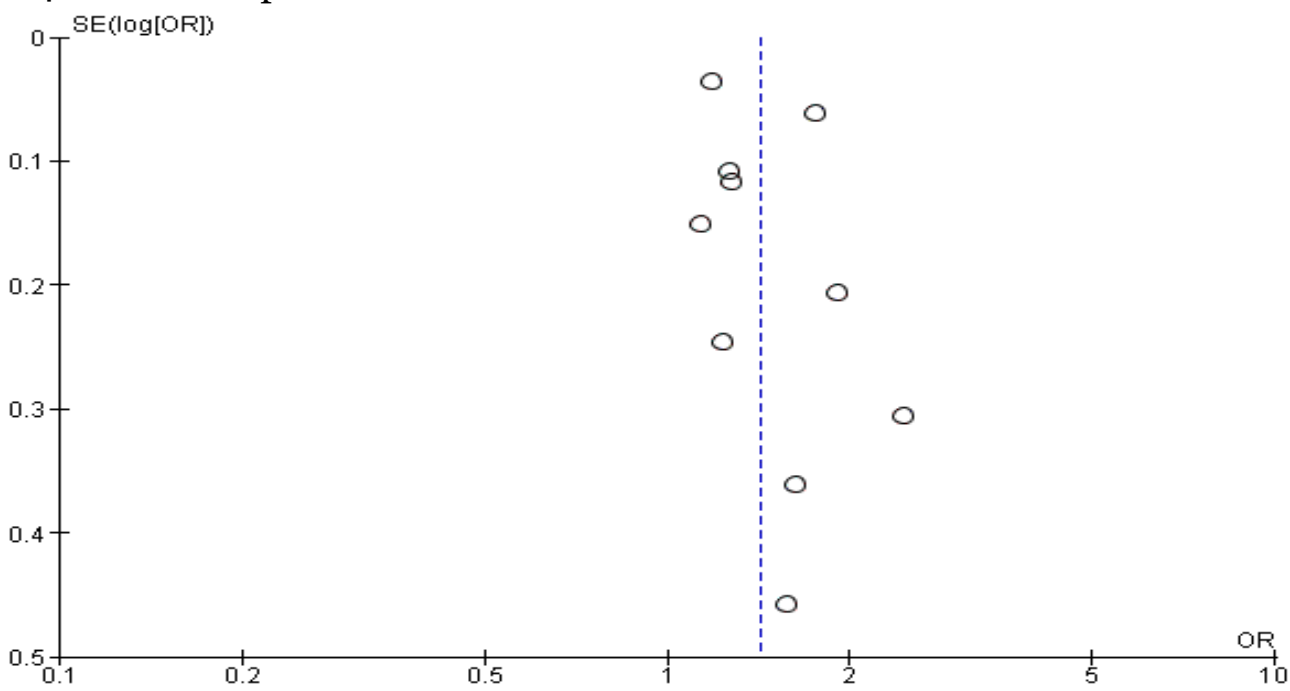

$(\mathrm{p}<0.001)$. The heterogeneity of the research data shows $\mathrm{I}^{2}=77 \%$ so that the distribution of the data is declared heterogeneous (random effect model).

Figure 6. Funnel plot of the relationship between sex and Covid-19 deaths

The funnel plot (Figure 6) shows a publication bias which is indicated by the plots on the right and left sides being symmetrical to each other and not forming an inverted funnel, where 5 plots are on the right and 5 plots are on the left. The plot on the left of the graph appears to have a standard error between 0 and 0.3 and the plot on the right has a standard error between 0 and 0.5 .

Table 5. Description of the primary studies of old age included in the meta-analysis

\begin{tabular}{|c|c|c|c|c|c|c|c|}
\hline $\begin{array}{l}\text { Author } \\
\text { (Year) }\end{array}$ & Country & $\begin{array}{l}\text { Study } \\
\text { Design }\end{array}$ & Sample & $\begin{array}{c}\mathbf{P} \\
\text { (Population) }\end{array}$ & $\begin{array}{c}\text { I } \\
\text { (Inter- } \\
\text { vention) }\end{array}$ & $\begin{array}{c}\mathrm{C} \\
\text { (Compa- } \\
\text { rison) }\end{array}$ & $\begin{array}{c}\text { O } \\
\text { (Out- } \\
\text { come) } \\
\end{array}$ \\
\hline $\begin{array}{l}\text { Hoobs et } \\
\text { al. (2021) }\end{array}$ & $\begin{array}{l}\text { United } \\
\text { States }\end{array}$ & $\begin{array}{l}\text { Retrospec- } \\
\text { tive Cohort } \\
\text { Study }\end{array}$ & 502 & $\begin{array}{l}\text { COVID-19 pati- } \\
\text { ents aged 65-79 } \\
\text { years }\end{array}$ & Young age & Old Age & $\begin{array}{l}\text { COVID-19 } \\
\text { deaths }\end{array}$ \\
\hline $\begin{array}{l}\operatorname{Imam} e t \\
\text { al. (2020) }\end{array}$ & $\begin{array}{l}\text { United } \\
\text { States }\end{array}$ & $\begin{array}{l}\text { Retrospec- } \\
\text { tive Cohort } \\
\text { Study }\end{array}$ & 1305 & $\begin{array}{l}\text { COVID-19 pati- } \\
\text { ents aged }>60 \\
\text { years }\end{array}$ & Young age & Old Age & $\begin{array}{l}\text { COVID-19 } \\
\text { deaths }\end{array}$ \\
\hline $\begin{array}{l}\text { Islam et } \\
\text { al. (2020) }\end{array}$ & $\begin{array}{l}\text { Bangla- } \\
\text { desh }\end{array}$ & $\begin{array}{l}\text { Spec- } \\
\text { ohort }\end{array}$ & 1016 & $\begin{array}{l}\text { COVID-19 pati- } \\
\text { ents aged }>60 \\
\text { years }\end{array}$ & Young age & Old Age & $\begin{array}{l}\text { COVID-19 } \\
\text { deaths }\end{array}$ \\
\hline $\begin{array}{l}\text { Leulseged } \\
\text { et } \quad \text { al. } \\
(2021)\end{array}$ & Ethiopia & $\begin{array}{l}\text { Retrospec- } \\
\text { tive Cohort } \\
\text { Study }\end{array}$ & 429 & $\begin{array}{l}\text { COVID-19 pati- } \\
\text { ents }>50 \text { years } \\
\text { old }\end{array}$ & Young age & Old Age & $\begin{array}{l}\text { COVID-19 } \\
\text { deaths }\end{array}$ \\
\hline $\begin{array}{l}\text { Yuhui et } \\
\text { al. (2021) }\end{array}$ & $\begin{array}{l}\text { China } \\
\text { (Asia) }\end{array}$ & $\begin{array}{l}\text { Retrospec- } \\
\text { tive Cohort } \\
\text { Study }\end{array}$ & 396 & $\begin{array}{l}\text { COVID-19 pati- } \\
\text { ents }>65 \text { years } \\
\text { old }\end{array}$ & Young age & Old Age & $\begin{array}{l}\text { COVID-19 } \\
\text { deaths }\end{array}$ \\
\hline $\begin{array}{l}\text { Cedano et } \\
\text { al. }(2020)\end{array}$ & $\begin{array}{l}\text { United } \\
\text { States of } \\
\text { America }\end{array}$ & $\begin{array}{l}\text { Retrospec- } \\
\text { tive Cohort } \\
\text { Study }\end{array}$ & 132 & $\begin{array}{l}\text { COVID-19 pati- } \\
\text { ents aged } 51-72 \\
\text { years }\end{array}$ & Young age & Old Age & $\begin{array}{l}\text { COVID-19 } \\
\text { deaths }\end{array}$ \\
\hline $\begin{array}{l}\text { Gerwen et } \\
\text { al. (2020) }\end{array}$ & $\begin{array}{l}\text { United } \\
\text { States of } \\
\text { America }\end{array}$ & $\begin{array}{l}\text { Retrospec- } \\
\text { tive Cohort } \\
\text { Study }\end{array}$ & 4,343 & $\begin{array}{l}\text { COVID-19 pati- } \\
\text { ents }>60 \text { years } \\
\text { old }\end{array}$ & Young age & Old Age & $\begin{array}{l}\text { COVID-19 } \\
\text { deaths }\end{array}$ \\
\hline
\end{tabular}


Permata et al./ Hypertension, Gender, Older Age, and COVID-19 Mortality

3. Results of the relationship between old age and covid-19 deaths

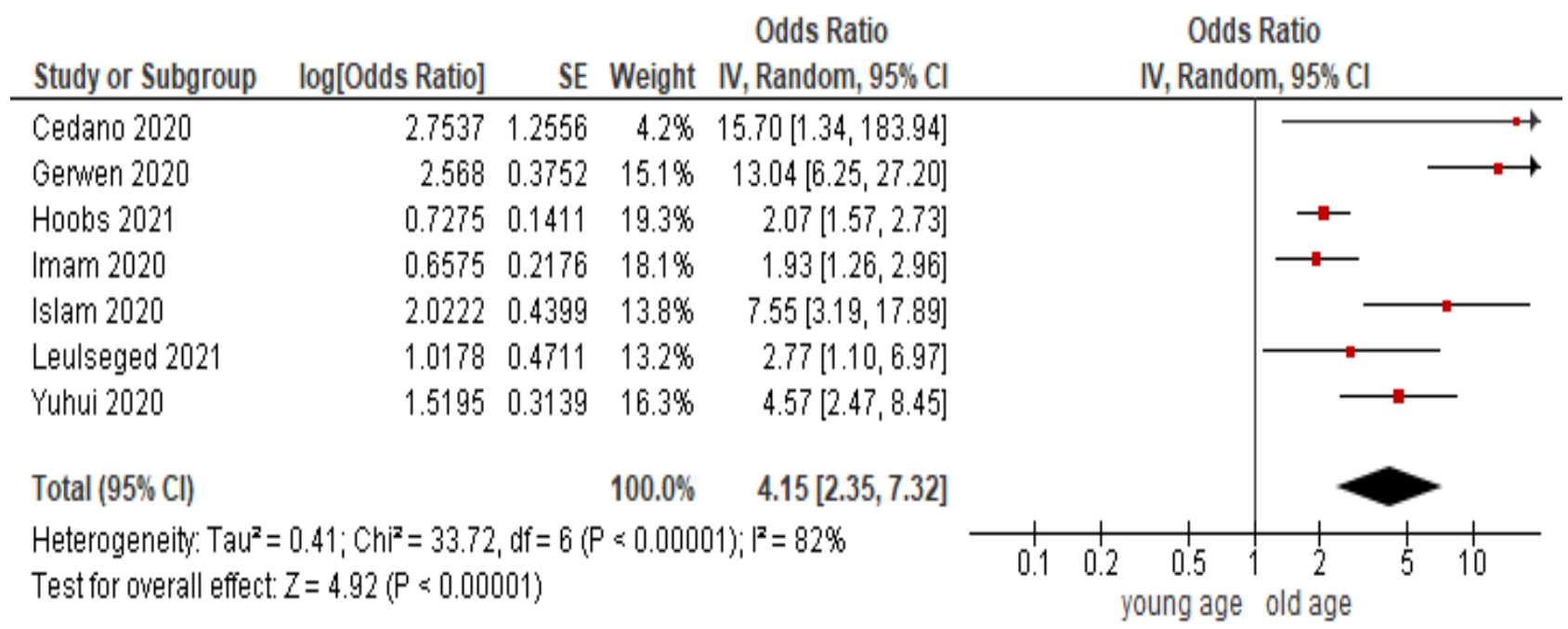

Figure 4. Forest plots the relationship between old age and Covid-19 mortality

Based on the results of the forest plot (Figure 4), the results of a meta-analysis of retrospective cohort studies show that old age increases mortality in COVID-19 patients. The results of a meta-analysis of retrospective cohort studies showed that old age increased the incidence of death in COVID19 patients by 4.15 times compared to young age $(\mathrm{p}<0.001)$. The heterogeneity of the research data shows $\mathrm{I}^{2}=82 \%$ so that the distribution of the data is declared heterogeneous (random effect model).

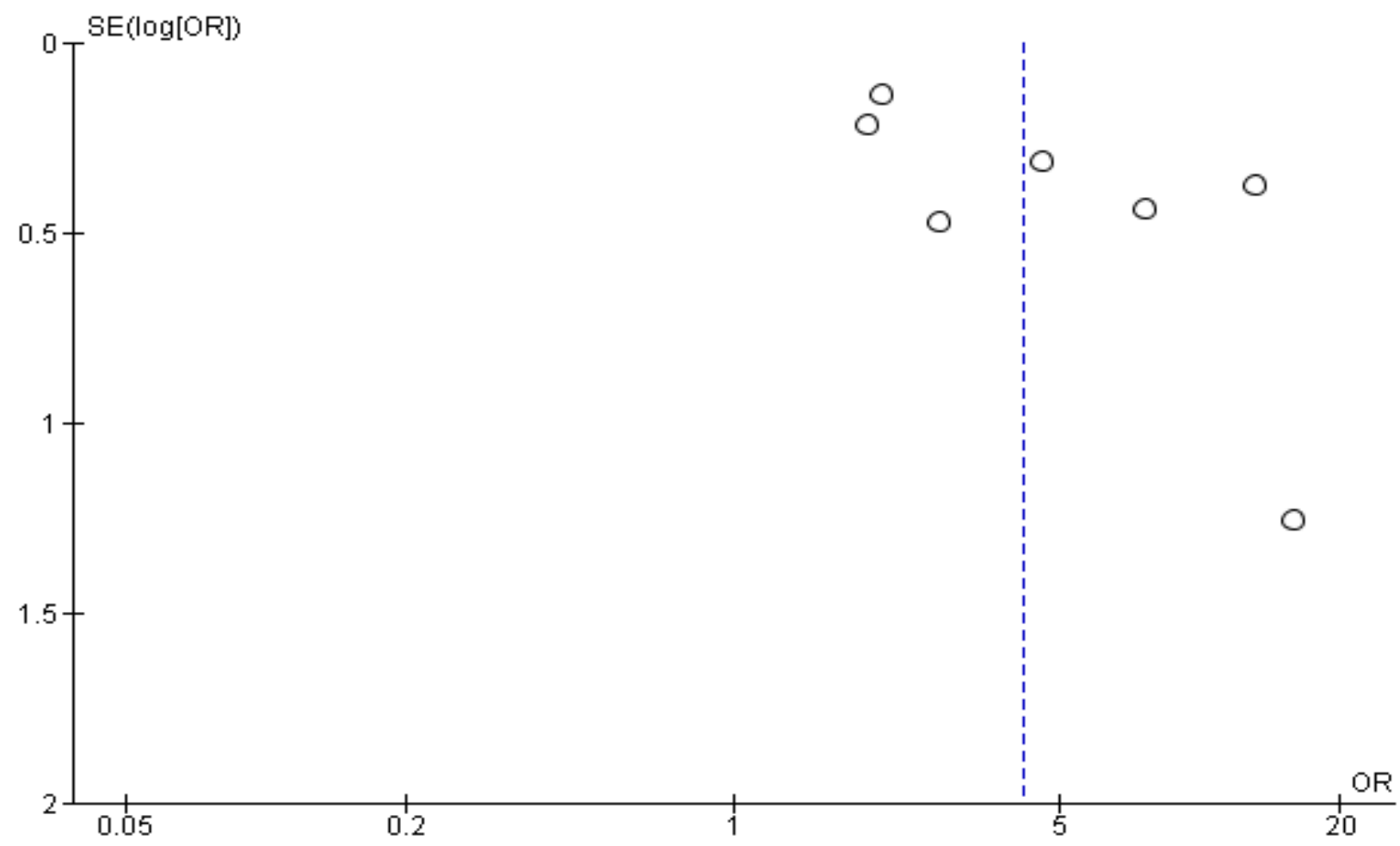

Figure 5. Funnel plot of the relationship between old age and Covid-19 deaths 
The funnel plot (Figure 5) shows a publication bias which is indicated by the plots on the right and left sides being symmetrical to each other and not forming an inverted funnel, where 4 plots are on the right and 4 plots are on the left. The plot on the left of the graph appears to have a standard error between 0 and 0.5 and the plot on the right has a standard error between 0 and 1.5 .

\section{DISCUSSION}

This systematic review and meta-analysis research raised the theme of the relationship between hypertension, gender and old age with COVID-19 mortality. This metaanalysis study uses research sources that control confounding factors or confounding factors that can be seen from the inclusion requirements of the study, namely multivariate analysis and the statistical results reported are adjusted odds ratio (aOR). The combined results of the relationship between hypertension, sex and old age with COVID-19 mortality were processed using the RevMan 5.3 application, while the results of the systematic study and metaanalysis were presented in the form of forest plots and funnel plots.

\section{The relationship between hyperten- sion and Covid-19 deaths}

The forest plot results in this meta-analysis using a retrospective cohort showed that hypertension was 1.40 times the risk of dying from COVID-19 compared to without hypertension and the results were statistically significant $(\mathrm{aOR}=1.40 ; 95 \% \mathrm{CI}=1.04$ to $1.89 ; \mathrm{p}=0.030$ ).

A similar study was conducted by Khan et al. (2020), who stated that hypertension had a 3.6 times risk of dying from COVID-19 $(\mathrm{OR}=3.6 ; 95 \% \mathrm{CI}=1.6-7.8 ; \mathrm{p}=$ 0.001). This is supported by the theory of angiotensin converting enzyme 2 (ACE-2) in the body, thereby increasing its expression and causing damage to alveolar cells.
These damaged alveolar cells in turn cause a series of inflammatory responses of the body that lead to a pattern of acute respiretory distress syndrome and even death. In line with this research, Darquenes et al. (2021) also stated that hypertension was 2.93 times the risk for COVID-19 death ( $\mathrm{aOR}=2.93$; 95\% $\mathrm{CI}=1.59-5.43 ; \mathrm{p}=0.0006)$.

Yuhui et al. (2020) mentioned that in a recent review, hypertension was considered as the main independent factor for poorer clinical outcome. hypertension alone contributes only to a relatively small degree to the development of severe infection, but not to death or the development of ARDS/ respiratory failure. Also, after ARDS/ respiratory failure developed hypertension in all investigated circumstances, severe respiretory failure may increase mortality in COVID-19 patients.

\section{Relationship between gender and Covid-19 deaths kematian}

The forest plot results in this meta-analysis using a retrospective cohort showed that male sex was at 1.42 times the risk of dying from COVID-19 compared to male female and the results were statistically significant $(\mathrm{aOR}=1.42 ; 95 \% \mathrm{CI}=1.20$ to $1.67 ; \mathrm{p}<0.001)$.

The results of this study are supported by Minhas et al. (2021), that the male sex is at risk for COVID-19 death, the female sex is at 2.04 times the risk of dying from COVID-19 compared to the male sex and the results are statistically significant $(\mathrm{aOR}=2.04 ; 95 \% \mathrm{CI}=1.44$ to $2.90 ; \mathrm{p}<$ 0.001).

Gupta et al. (2020) in their study stated that the male sex was at risk of 1.96 times for COVID-19 death compared to the female sex and the results were statistically significant $(\mathrm{OR}=1.96 ; 95 \% \mathrm{CI}=1.34$ to $2.90 ; \mathrm{p}=0.001)$. These results are consistent with the number of $\mathrm{X}$ chromosomes containing many genes that code for the modulation of immune function. Fe males 
have a higher proportion of this immuneboosting Mgen which is usually protective. Other things like increasing the expression and activation of angiotensin type 2 (AT2) receptors in women lead to a stronger antiinflammatory immune response.

\section{Relationship between old age and Covid-19 death}

The forest plot results in this meta-analysis using a retrospective cohort showed that older people were 4.15 times more likely to die from COVID-19 compared to young people and the results were statistically significant $(\mathrm{aOR}=4.15 ; 95 \% \mathrm{CI}=2.35$ to 7.32; $\mathrm{p}<0.001)$.

Chung et al. (2020) in their study stated that old age has a 7.10 times risk of dying from COVID-19 compared to young people and the results are statistically significant $(\mathrm{OR}=7.10 ; 95 \% \mathrm{CI}=1.82$ to 27.6 ; $\mathrm{p}=0.005)$. This study is in line with Gupta et al. (2020) in their study stated that old age is at risk of 1.02 times for COVID-19 death compared to young age and the results are statistically significant $(\mathrm{OR}=$ 1.02; 95\% $\mathrm{CI}=1.01$ to $1.04 ; \mathrm{p}=<0.001$ ).

A similar study on the association of old age with COVID-19 mortality was conducted by Khan et al. (2020), who stated that age was 8.3 times more likely to die from COVID-19 compared to younger age and the results were statistically significant $(\mathrm{OR}=8.3 ; 95 \% \mathrm{CI}=1.1$ to $63.1 ; \mathrm{p}=$ 0.040). Additional findings in our study suggest that the risk of death is higher among older patients with a higher risk among older adults similar to global trends. Other reasons suggested for higher mortality include behavioral, social and biological differences. .

Klang et al. (2020) stated that old age is 1.7 times the risk 7.10 times for COVID19 death. In immunopathology, susceptibility to infection in the elderly is usually explained by immunosenescence. Innate immune cells are impaired as a result, cells involved in innate immunity are not activated efficiently during infection, and progression to an adaptive immune response does not occur in a coordinated manner. These changes reduce the effectiveness of viral clearance and increase the likelihood of triggering an aberrant immune response in which cytokines are extensively released by activated immune cells, generating a cytokine storm. Another well-known feature of aging immunity is chronic subclinical systemic inflammation, also known as inflammation. Inflammation is the main pathogenic mechanism in COVID-19. Therefore, inflammation is thought to contribute to poorer outcomes in elderly patients with COVID-19.

The limitation of this study is that there is a publication bias shown in the funnel plot of the case control study in the variables of gender and old age. There is a language bias, because in this study the selected articles were only published in English, thus ignoring articles in other languages. Search bias because in this study, researchers only used 4 databases (PubMed, Science Direct, Springer Link, and PMC Europe) thus ignoring other search sources.

\section{AUTHOR CONTRIBUTION}

Annissa is the main researcher who selects the topic, searches and collects research data. Bhisma Murti and Didik G Tamtomo played a role in analyzing data and reviewing research documents.

\section{FUNDING AND SPONSORSHIP}

This study is self-funded.

CONFLICT OF INTEREST

There is no conflict of interest in this study. 


\section{ACKNOWLEDGEMENT}

We are very grateful to database providers PubMed, Science Direct, Springer Link and Europe PMC.

\section{REFERENCES}

Buzan, Tony (2020). Mind Mapping. https://www.tonybuzan.com/mindmapping/diakses pada 01/06/2020.

Borgne P, Morgane S, François S, Hamid M, Yvon R, Karine A, Eric B, et al. (2020). SARS-CoV-2 viral load in nasopharyngeal swabs in the emergency department does not predict COVID-19 severity and mortality. Acad Emerg Med. 28: 306-313.

CDC (2020). Case, Data and Survailance: COVID-19. Vailable from: http://www.cdc.gow/cprpnavirus/2021-ncov/cases-update/index.html Diakses pada tanggal 6 Februari 2021.

CEBMa (2014). Critical Appraisal. CDC (2020). Case, Data and Survailance: COVID-19. Vailable from: http://www.cdc.gow/cprpnavirus/2021ncov/cases-update/index.html

Diakses pada tanggal 6 Februari 2021

Cedano J, Emilio F, Melissa G, Melvin S, Islam Y, Sarah A, Andrew K, et al. (2021). Characteristics and outcomes of patients with COVID-19 in an intensive care unit of a community hospital; retrospective cohort study. J Community Hospital Internal Med Perspectives. 11(1): 27-32. http://doi.org/10.1080/200009666.2020.18305 16020.1830516

Chung SM, Yin Y, Eunyeong, Ji S, Kyu C, Hyoung W, Jian Hur, et al. (2020). The risk of diabetes on clinical outcomes in patients with Coronavirus Disease 2019: A Retrospective Cohort Study. Diabetes Metab J. 44: 405-413. pISSN 233-6079 • eISSN 233-6087.
Dehingia N (2020). Sex differences in COVID-19 case fatality: Do we know enough?. The Lancet Religion Hearth. o9: E14-E15. http://doi.org/10.1016/S214-109X(20)30464-2.

Elimian KO, Ochu CL, Ebhodaghe B, et al. (2020). Patient characteristics associated with COVID-19 positivity and fatality in Nigeria: retrospective cohort study. BMJ Open 10: e044079. doi:10.1136/ bmjopen-2020-044079

Gerwen M, Mathilda A, Christine L, Joshua B, Eric G, Leonard N, Douglas T, et al. (2020). Risk factors and outcomes of COVID-19 in New York City; a retrospective cohort study. J Med Virol. 93: 907-915. doi: 10.1002/jmv.26337

Gupta R, Raag A, Zaheer B, Absia J, Donghai W, John D, Mohamed A, et al. (2021). Higher comorbidities and early death in hospitalized AfricanAmerican patients with Covid-19. BMC Infectious Disease. (2021) 21:78. https://doi.org/10.1186/s12879-02105782-9

Guzman P, Anna D, Sujit M, Peter C, Roberta F, Mara D, Alessandra L, et al. (2020). Clinical characteristics and predictors of outcomes of hospitalized patients with Coronavirus Disease 2019 in a Multiethnic London National Health Service Trust: A retrospective cohort study. Clin Infect Dis. doi: 10.1093/cid/ciaa1091.

Harrison SL, Fazio-Eynullayeva E, Lane DA, Underhill P, Lip GYH (2020). Comorbidities associated with mortality in 31,461 adults with COVID-19 in the United States: A federated electronic medical record analysis. PLoS Med 17(9): e1003321. https://doi.org/10.1371/journal. pmed.100332.

Hashemi N, Kathleen V, Walker R, Joyce C, Thomas R, Bazarbashi AN, Kelly E, et 
al. (2020). Impact of chronic liver disease on outcomes of hospitalized patients with COVID-19: A multicentre United States experience. Liver International. 1-7. Doi: 10.1111/liv.1458

Hobbs A, Pharm, Turner N, Imad O, Morgan K, Ronald M, Muhammad S (2020). Risk factors for mortality and progression to severe COVID-19 disease in the Southeast United States (US): A Report from the SEUS Study Group. Infect Control Hosp Epidemiol. 1-33 https://doi.org/10.1017/ice.2020.1435.

Imam Z, Odish F, Gill I, O'Connor D, Armstrong J, Vanood A, Ibironke O, Hanna A, Ranski A, Halalau A (2020). Older age and comorbidity are independent mortality predictors in a large cohort of 1305 COVID-19 patients in Michigan, United States. J Intern Med. 288(4): 469-476. https://doi.org/10.1111/joim.13119.

Islam MZ, Riaz BK, Islam ANMS, Khanam F, Akhter J, Choudhury R, Farhana N, Jahan NA, Uddin MJ, Efa SS (2020). Risk factors associated with morbidity and mortality outcomes of COVID-19 patients on the 28th day of the disease course: a retrospective cohort study in Bangladesh. Epidemiol Infect. 148:e263. https://doi.org/10.1017/So950268820002630.

Kang S, Sook IJ (2020). Age-related morbidity and mortality among patients with COVID-19. Infect Chemother. 52(2): 154-164. https://doi.org/10.3947/ic.2020.52.2.154.

Khan MS, Dogra R, Miriyala LKV, Salman FNU, Ishtiaq R, Patti DK, et al. (2021) Clinical characteristics and outcomes of patients with Corona Virus Disease 2019 (COVID-19) at Mercy Health Hospitals, Toledo, Ohio. PLoS ONE
16(4): e0250400. https://doi.org/10.1371/journal. pone.0250400.

Leulseged TW, Hassen IS, Ayele BT, Tsegay YG, Abebe DS, Edo MG, et al. (2021) Laboratory biomarkers of COVID-19 disease severity and outcome: Findings from a developing country. PLoS ONE. 16(3): e0246087. https://doi. org/10.1371/journal.pone.0246087

Magleby R, Lars F, Alex T, Matthew S, Mangala R, Joel P, Parag G, et al. (2020). Impact of severe acute respiratory syndrome Coronavirus 2 viral load on risk of intubation and mortality among hospitalized patients with coronavirus disease 2019. Clin Infect Dis. 20(20): 1-9. Doi: 10.1093/cid/ciaa851.

Manuel J, Verónica B, Michele R, Jesica M, Diana P, María C, Manel R, et al. (2020). Clinical Characteristics and Risk Factors for Mortality in Very Old Patients Hospitalized With COVID-19 in Spain. J Gerontol A Biol Sci Med Sci, 10(10): 1-10. doi 10.1093/Gerona/glaa243.

Minhas A, Julie K, Sung-Min C, Erin D, Thomas M, Nisha A, Garima S, et al. (2021). The role of sex and inflamemation in cardiovascular outcomes and mortality in COVID-19. IJCA29540. https://doi.org/10.1016/j.ijcard.2021.05.011.

Musharahat P (2020). Prevalence and associated risk factors of mortality among COVID-19 patients: A Meta-Analysis. J Community Health. 45: 1270-1282. https://doi.org/10.1007/s10900-020o0920-x.

Nasir G, Giovanni R, Silvio B (2020). Case fatality rate and charateristic of patients dying in relation to COVID-19 in Italy. JAMA. 323(18): 1775-1776. doi: 10.1001/jama.2020.4683 
Osibogun A, Balogun M, Abayomi A, Idris J, Kuyinu Y, Odukoya O, et al. (2021) Outcomes of COVID-19 patients with comorbidities in southwest Nigeria. PLoS ONE. 16(3): e0248281. https://doi.org/10.1371/journal.pone.024828 1.

Magleby R, Westblade LF, Trzebucki A, Simon MS, Rajan M, Park J, Goyal P, Safford MM, Satlin MJ (2020). Impact of SARS-CoV-2 viral load on risk of intubation and mortality among hospitalized patients with Coronavirus disease 2019. Clin Infect Dis. https://dx.doi.org/10.1093\%2Fcid\%2Fciaa851.

Rosa FG, Palazzo A, Rosso T, Shbaklo N, Mussa M, Boglione L, Borgogno E, Rossati A, Mornese P, Scabini S, et al. (2021). Risk factors for mortality in COVID-19 hospitalized patients in Piedmont, Italy: Results from the multicenter, regional, CORACLE Registry. J Clin Med. 10: 1951. https://doi.org/10.3390/jcm10091951.

Rosenthal N, Zhun C, Jake G, Jim S, Pharm, Stella S (2020). Risk Factors Associated with In-Hospital Mortality in a US National Sample of Patients with COVID-19. Jama Network Open. 3(12): e2029058. Doi: 10.1001/jamanetworkopen. 2020.29058.

Surendra R, Iqbal RF, Bimandra A, Lenny L, Kartika S, Verry A, Widyastuti.
(2020). Clinical characteristics and mortality associated with COVID-19 in Jakarta, Indonesia: A hospitalbased retrospective cohort study. Elsevier. https://doi.org/10.1016/j.lanwpc.2021.100108

Walker J, Dolly S, Ng L, Prior-Ong M, Sabapathy K (2020) The role of CPAP as a potential bridge to invasive ventilation and as a ceiling-ofcare for patients hospitalized with Covid-19-An observational study. PLoS ONE 15(12): eo244857. https://doi.org/10.1371/journal.pone.0244857

Yip TCF, Lui GCY, Wong VWS, Chow VCY, Ho THY, Li TCM, Tse YK, et al. (2021). Liver injury is independently associated with adverse clinical outcomes in patients with COVID-19. Gut Gut. 70(4): 733-742. https://doi.org/10.1136/gutjnl-2020-321726.

Yuhui S, Xizhou G, Lijing J, Ning X, Liuquan C, Bo L, Sibing Z, et al. (2020). Independent and combined effects of hypertension and diabetes on clinical outcomes in patients with COVID-19: A retrospective cohort study of Huoshen Mountain Hospital and Guanggu Fangcang Shelter Hospital. J Clin Hypertens. 23: 218-231. Doi: 10.1111/jch.14146. 